\title{
Association of lopinavir concentrations with plasma lipid or glucose concentrations in HIV-infected South Africans: a cross sectional study
}

\author{
Phumla Z Sinxadi ${ }^{1 *}$, Helen M Mcllleron ${ }^{1}$, Joel A Dave ${ }^{2}$, Peter J Smith ${ }^{1}$, Naomi S Levitt ${ }^{2}$ and Gary Maartens ${ }^{1}$
}

\begin{abstract}
Background: Dyslipidaemia and dysglycaemia have been associated with exposure to ritonavir-boosted protease inhibitors. Lopinavir/ritonavir, the most commonly used protease inhibitor in resource-limited settings, often causes dyslipidaemia. There are contradictory data regarding the association between lopinavir concentrations and changes in lipids.
\end{abstract}

Aim: To investigate associations between plasma lopinavir concentrations and lipid and glucose concentrations in HIV-infected South African adults.

Methods: Participants stable on lopinavir-based antiretroviral therapy were enrolled into a cross-sectional study. After an overnight fast, total cholesterol, triglycerides, and lopinavir concentrations were measured and an oral glucose tolerance test was performed. Regression analyses were used to determine associations between plasma lopinavir concentrations and fasting and 2 hour plasma glucose, fasting cholesterol, and triglycerides concentrations.

Results: There were 84 participants (72 women) with a median age of 36 years. The median blood pressure, body mass index and waist: hip ratio were $108 / 72 \mathrm{mmHg}, 26 \mathrm{~kg} / \mathrm{m}^{2}$ and 0.89 respectively. The median CD4 count was $478 \mathrm{cell} \mathrm{s} / \mathrm{mm}^{3}$. Median duration on lopinavir was 18.5 months. The median (interquartile range) lopinavir concentration was 8.0 (5.2 to 12.8) $\mathrm{\mu g} / \mathrm{mL}$. Regression analyses showed no significant association between lopinavir pre-dose concentrations and fasting cholesterol ( $\beta$-coefficient $-0.04(95 \% \mathrm{Cl}-0.07$ to 0.00$)$ ), triglycerides ( $\beta$-coefficient -0.01 ( $95 \% \mathrm{Cl}-0.04$ to 0.02)), fasting glucose ( $\beta$-coefficient -0.01 ( $95 \% \mathrm{Cl}-0.04$ to 0.02$)$ ), or 2-hour glucose concentrations ( $\beta$-coefficient $-0.02(95 \% \mathrm{Cl}-0.09$ to 0.06$)$ ). Lopinavir concentrations above the median were not associated with presence of dyslipidaemia or dysglycaemia.

Conclusions: There was no association between lopinavir plasma concentrations and plasma lipid and glucose concentrations.

Keywords: Lopinavir, Hypercholesterolaemia, Hypertriglyceridaemia, Impaired glucose metabolism, Antiretroviral therapy, Pharmacokinetics

\section{Introduction}

Ritonavir-boosted lopinavir (LPV/r) is a potent protease inhibitor (PI) widely used in a second-line antiretroviral regimens in low and middle-income countries [1]. Treatment with $\mathrm{LPV} / \mathrm{r}$ has been associated with the development of dyslipidaemia [2,3], which is a known risk factor

\footnotetext{
*Correspondence: phumla.sinxadi@uct.ac.za

'Department of Medicine, Division of Clinical Pharmacology, University of Cape Town, Cape Town, South Africa

Full list of author information is available at the end of the article
}

for cardiovascular complications. The Women's Interagency HIV study showed that dyslipidaemia was more severe in HIV infected women on ritonavir-boosted PI containing antiretroviral therapy (ART), compared with untreated HIV infected women or non-PI based ART [4]. There are conflicting results regarding an association between LPV/r use and the development of insulin resistance and new onset diabetes [5-9]. Furthermore, there are contradictory data as to whether the metabolic toxicity is associated with higher plasma concentrations

\section{Biomed Central}


of either lopinavir or ritonavir [10-12]. Widespread longterm PI use may be limited by these metabolic complications. Data regarding an association between plasma lopinavir and plasma glucose concentration are lacking.

The aim of our study was to investigate whether there is an association between plasma lopinavir and plasma lipids and glucose concentrations. We hypothesized that higher plasma lopinavir concentrations would be associated with higher prevalence of dyslipidaemia and dysglycaemia.

\section{Materials and methods}

\section{Study design and participants}

We conducted a prospective cross sectional study between February 2007 and January 2008. Consecutive ambulatory HIV infected African adults who presented for a routine follow up visit at primary and tertiary level clinics in Cape Town were recruited. Participants were eligible if they were on lopinavir-based therapy for a minimum of six months. Participants with renal or hepatic disease, active opportunistic infection, diabetes, history of diabetes or dyslipidaemia and self-reported non-adherence were excluded. The study was approved by the University of Cape Town research ethics committee.

\section{Clinical and laboratory evaluations}

After obtaining informed consent, we instructed the participants to undergo an overnight fast and to note the time of taking the evening dose of lopinavir on the day proceeding the study day. On the study day, participants underwent an oral glucose tolerance tests (OGTT). Blood was drawn at 0 and 120 minutes after ingestion of $75 \mathrm{~g}$ glucose in $250 \mathrm{ml}$ water, into heparinised or sodium fluoride tubes as appropriate, and kept on ice until centrifugation within 4 hours. Plasma lopinavir concentrations and serum fasting glucose, cholesterol, and triglyceride concentrations were determined from the 0 minute samples of the OGTT. The morning doses of ART were given after 2 hour glucose samples were taken.

Lopinavir drug concentrations were analyzed by fully validated methods using liquid chromatography-tandem mass spectrometry (LC-MS/MS) on an Applied Biosystems MDS Sciex API 4000 tandem mass spectrometer at our ISO17025 compliant and accredited analytical laboratory as previously described [13]. The assay range was 0.05 to $20 \mu \mathrm{g} / \mathrm{mL}$. Accuracy ranged from 94 to $103 \%$. Any samples with lopinavir concentrations below the limit of quantification $(0.05 \mu \mathrm{g} / \mathrm{mL})$ were fixed to 0.025 $\mu \mathrm{g} / \mathrm{mL}$. Serum glucose and lipid concentrations were determined by standard methods using the ACE Alera Clinical Chemistry System (Alfa Wassermann Diagnostic Technologies, Woerden, Netherlands). Impaired fasting glucose (IFG) and impaired glucose tolerance (IGT) were defined according to the American Diabetes Association criteria [14]. Hypercholesterolaemia and hypertriglyceridaemia were defined according to the NCEP III criteria [15].

We reviewed medical records to determine duration on antiretroviral therapy, viral load and CD4 counts. Self reported adherence was determined using a validated 4day adherence questionnaire administered by trained field workers [16]. Basic anthropometric measurements were measured by a biokineticist.

\section{Pharmacokinetic and statistical analysis}

The plasma lopinavir concentrations were obtained from 0 minute of the OGTT. For data points below the limit of quantification $(0.05 \mu \mathrm{g} / \mathrm{mL})$, the lopinavir concentrations were fixed to $0.025 \mu \mathrm{g} / \mathrm{mL}$. The associations between lopinavir concentrations and glucose, triglycerides, and cholesterol were determined using univariate linear regression and multivariate analyses. Logistic regression was used to determine associations between lopinavir concentrations above the median and metabolic complications (hypercholesterolaemia, hypertriglyceridaemia and dysglycaemia).

\section{Sample size calculation}

A previous study found a positive correlation (rho) of 0.32 between lopinavir trough levels and triglyceride levels [10]. When the sample size is 50, the linear regression test of rho $=0$ (alpha $=0.050$ two-sided) for normally distributed triglyceride concentrations, will have $80 \%$ power to detect a rho of 0.375 .

\section{Results}

Of 93 participants enrolled for the study, nine were excluded due to missing anthropometric and metabolic data or inaccurate dose-sampling time. The baseline characteristics of the 84 participants included in the analysis are in Table 1 . The majority were women, reflecting the epidemic seen in our clinical practice. All participants reported $100 \%$ adherence. The prevalence of hypercholesterolaemia, hypertriglyceridaemia, impaired fasting glucose, impaired glucose tolerance, and diabetes were $29 \%, 29 \%, 25 \%, 14 \%$ and $4 \%$ respectively. The plasma LPV concentrations are shown in Figure 1. The median (IQR) time after dose was 13.2 (12.6 to 14.1) hours. There were ten participants whose lopinavir concentrations were below $1 \mu \mathrm{g} / \mathrm{mL}$ (12\%). Of these, 4 were virologically suppressed, 4 had virologic failure, and 2 had missing viral load data. As there was a significant association between time after dose and lopinavir concentrations, the time after dose was included in the multivariate regression analyses investigating the association between lopinavir concentrations and metabolic parameters. There were no significant associations between lopinavir concentrations and lipid and glucose 
Table 1 Study population characteristics, $\mathrm{N}=\mathbf{8 4}$

\begin{tabular}{|c|c|}
\hline Variable & $\begin{array}{l}\text { Median (IQR) } \\
\text { or } \mathrm{n} / \mathrm{N}(\%)\end{array}$ \\
\hline Age (years) & $36(32-42)$ \\
\hline Female n/N (\%) & $72 / 84(86)$ \\
\hline \multicolumn{2}{|l|}{ Race n/N (\%) } \\
\hline Black & $84 / 84(100)$ \\
\hline Weight (kg) & $69(60-82)$ \\
\hline Body mass index $\left(\mathrm{kg} / \mathrm{m}^{2}\right)$ & $26(23-32)$ \\
\hline Waist: hip ratio & $0.88(0.82-0.94)$ \\
\hline \multicolumn{2}{|l|}{ Skin fold thickness (mm) } \\
\hline Triceps & $17(11-25)$ \\
\hline Abdomen & $24(17-40)$ \\
\hline Thigh & $30(17-46)$ \\
\hline Calf & $16(9-21)$ \\
\hline Blood pressure mmHg & 108/72 (102/66-119/77) \\
\hline \multicolumn{2}{|l|}{ CD4 count (cells/ mm³) } \\
\hline Pre-ART & $103(37-140)$ \\
\hline Current & $468(291-623)$ \\
\hline \multicolumn{2}{|l|}{ Current viral load } \\
\hline Proportion with $<400$ copies $/ \mathrm{mL}(\%)^{*}$ & $64 / 74(86)$ \\
\hline Duration on lopinavir (months) & $19(9-29)$ \\
\hline \multicolumn{2}{|l|}{ Concurrent ART n/N (\%) } \\
\hline Zidovudine/didanosine & $51 / 84(61)$ \\
\hline Zidovudine/lamivudine & $17 / 84(20)$ \\
\hline Stavudine/lamivudine & $10 / 84(12)$ \\
\hline Efavirenz & 4/84 (5) \\
\hline Nevirapine & 2/84 (2) \\
\hline \multicolumn{2}{|l|}{ Metabolic parameters (mmol/L) } \\
\hline Fasting cholesterol & 4.3 (3.7 to 5.3 ) \\
\hline Fasting triglycerides & $1.3(0.9$ to 1.8$)$ \\
\hline Fasting glucose & $5.2(4.7$ to 5.7$)$ \\
\hline 2 hour glucose & $6.3(5.4$ to 8.1$)$ \\
\hline
\end{tabular}

* 10 participants had missing current viral load data.

concentrations on simple regression analyses, or after adjusting for age, sex, time after dose, and duration on lopinavir (Table 2). The lack of association between lopinavir concentrations and the metabolic parameters persisted when the 10 participants with lopinavir concentrations below $1 \mu \mathrm{g} / \mathrm{mL}$ (as they were probably non-adherent) were excluded (data not shown). Sixty one percent of participants were on zidovudine and didanosine combination (Table 1), as recommended by the South African antiretroviral guidelines at the time. However, the different NRTI backbone was not associated with any of the outcomes evaluated. For example, for triglycerides, in the participants of the same age, sex,

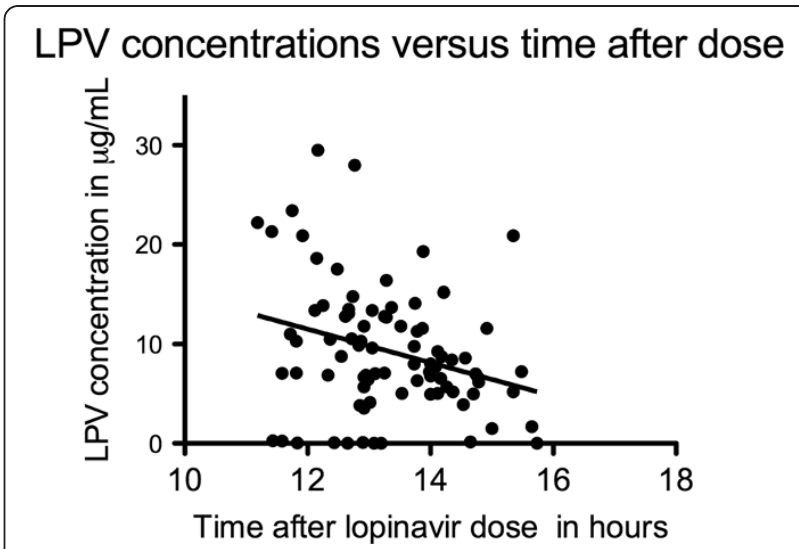

Figure 1 Scatter plot of lopinavir concentrations $(\mu \mathrm{g} / \mathrm{mL})$ and the time after the last lopinavir dose (hours). The black dots denote individual lopinavir concentrations plotted against the time after the last unobserved lopinavir dose (dose to sampling time) from 84 participants. The regression line shows negative correlation between the lopinavir concentrations and the dose to sampling time (Spearman rho $(95 \% \mathrm{Cl})=-0.28(-0.47$ to -0.06$)$, $\mathrm{p}$-value 0.01$)$.

duration on lopinavir, and with same lopinavir concentration taken at the same time after dose, there was no significant difference between those who were on zidovudine/didanosine combination compared to other NRTI combinations [mean difference $-0.22(-0.65$ to $0.21) \mathrm{mmol} / \mathrm{L}$, $\mathrm{p}$ value $=0.31]$. Similarly, there was no association with NRTI backbone and cholesterol if there all parameters remained the same [mean difference $-0.18(-0.73$ to 0.37$) \mathrm{mmol} / \mathrm{L}, \mathrm{p}$ value $=0.51$ ]. There was no association between lopinavir concentrations above the median $(8 \mu \mathrm{g} / \mathrm{mL})$ and dyslipidaemia or dysglycaemia (see Figure 2).

\section{Discussion}

To our knowledge, this is the first study to investigate the association between lopinavir concentrations and serum glucose concentrations, and the first to investigate associations between lopinavir concentrations and serum lipids in a South African population. Despite a high prevalence of dyslipidaemia (29\%) and dysglycaemia (42\%) in 84 black South African HIV-infected adults treated with ritonavir-boosted lopinavir for a median duration of 19 months, we found no association between plasma lopinavir concentrations and lipid or glucose concentrations. There was also no significant association between the lopinavir concentrations above the median, and hypercholesterolaemia, hypertriglyceridaemia or dysglycaemia.

We found the median lopinavir concentration was 8 $\mu \mathrm{g} / \mathrm{mL}$, which is higher than reported elsewhere $[17,18]$, but comparable to the trough concentrations after observed doses in a study conducted by our group from the same community [19]. Lopinavir pharmacokinetics 
Table 2 Association between lopinavir trough concentrations and lipid and glucose concentrations

\begin{tabular}{|c|c|c|c|c|}
\hline \multirow[b]{2}{*}{ Variable } & \multicolumn{2}{|c|}{ Univariate analyses } & \multicolumn{2}{|c|}{ Multivariate analyses } \\
\hline & Beta coefficient $(95 \% \mathrm{Cl})$ & $P$ value & Beta coefficient $(95 \% \mathrm{Cl})$ & $P$ value \\
\hline Total cholesterol & $-0.04(-0.07$ to 0.00$)$ & 0.07 & $-0.02(-0.06$ to 0.01$)$ & 0.21 \\
\hline Triglycerides & $-0.01(-0.04$ to 0.02$)$ & 0.53 & $-0.00(-0.03$ to 0.02$)$ & 0.86 \\
\hline Fasting glucose & $-0.01(-0.04$ to 0.02$)$ & 0.44 & $-0.00(-0.03$ to 0.02$)$ & 0.78 \\
\hline 2 hour glucose & $-0.02(-0.09$ to 0.06$)$ & 0.64 & $0.00(-0.05$ to 0.06$)$ & 0.85 \\
\hline
\end{tabular}

Adjusted for time after dose, age, sex and duration on lopinavir. In the multivariate model for 2 hour glucose, time after dose was an independent predictor [ $\beta$-coefficient 0.56 (95\% Cl 0.23 to 0.88$), p=0.001]$.

demonstrate considerable interindividual variability, which may affect treatment outcomes. At least part of this variability may be explained by host genetic factors. Associations between human genetic variants and lopinavir exposure are incompletely understood and need to be explored.

The lack of an association between lopinavir concentrations and lipid or glucose concentrations that we found can be explained as follows: First, like all protease inhibitors, lopinavir exerts its antiviral activity intracellularly, and the plasma and intracellular half-lives are different [20]. However, the positive correlation between lopinavir plasma and intracellular concentrations reported at 4 weeks was not sustained at 24 weeks of treatment [20]. More importantly, the mechanism of toxicity associated with protease inhibitor use, including lopinavir, is still poorly understood, but is thought to be related to interference with some cellular endogenous processes. For example, lopinavir binds to lipoprotein receptor related protein (LPR), impairing hepatic chylomicron uptake and triglyceride clearance by LPR -lipoprotein lipase complex, causing hyperlipidaemia [21]. In susceptible individuals, the resulting hyperlipidaemia may induce diabetes [21]. Second, clinical manifestations of LPV/r toxicity are also influenced by host susceptibility such as age, sex, weight, race, advanced HIV disease, concomitant ART, higher baseline triglyceride or cholesterol concentrations, and genetic susceptibility [2,22,23]. Finally, we hypothesize that the if the association between plasma lopinavir concentrations and toxicity exists, it exists in much lower concentrations, as the dose-response curve is likely to be flat at the concentrations found in our study. Therefore, the influence of lopinavir plasma concentrations on lipids at therapeutic doses, is likely to be small and larger studies are needed to detect the small differences. Furthermore, recent data suggests that lopinavir does not impair insulin sensitivity [7,8], and therefore, the lack of an association between lopinavir and glucose concentrations is not surprising.

Our findings are in contrast with findings from a small study conducted in 19 patients, which reported that

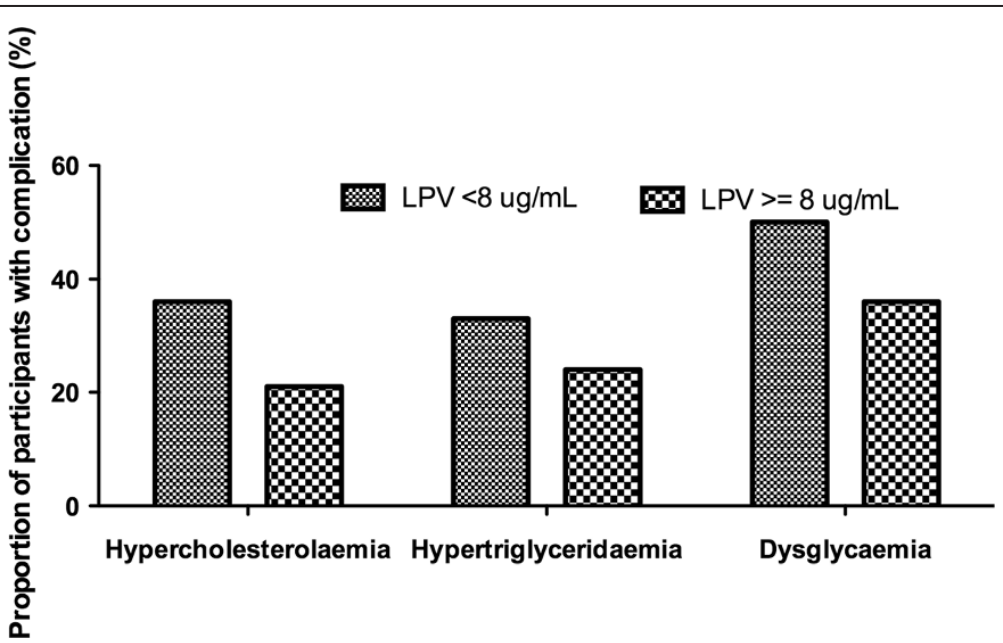

Figure 2 Proportion of participants with dyslipidaemia and dysglycaemia categorized by the lopinavir concentration below or above the median $(8 \mu \mathrm{g} / \mathrm{mL})$. The bar graphs show the proportion of participants (\%) who had hypercholesterolaemia, hypertriglyceridaemia and dysglycaemia in 42 participants with lopinavir below the median (fine checked bars) and in 42 participants with lopinavir concentrations equal to, or above the median (course checked bars). There was no association between the lopinavir concentrations above the median and the metabolic complications. The odds ratios for lopinavir above the median and the complications were: hypercholesterolaemia OR (95\%Cl): 0.49 (0.19 to 1.30); hypertriglyceridaemia OR (95\%Cl): 0.56 (0.29 to 1.33); dysglycaemia OR (95 Cl): 0.63 (0.24 to 1.63). 
lopinavir trough concentrations were higher in three patients experiencing grade 3 or 4 lipid elevations [11]. A second larger study $(\mathrm{n}=126)$ found that patients with fasting triglyceride concentrations above the median had higher lopinavir trough concentrations, but no correlation was found between lopinavir and cholesterol concentrations [10]. Four other studies reported findings similar to ours, with no association between lopinavir and lipid concentrations $[12,17,24,25]$. The older protease inhibitor indinavir is known to cause diabetes [5]. However, after 12 months of treatment with lopinavir, none of the 73 patients included in another study developed diabetes [6]. Studies in healthy volunteers have shown that insulin sensitivity wasn't altered by lopinavir in healthy HIV negative men $[7,8]$. However, a single dose study in healthy volunteers showed that lopinavir could inhibit glucose uptake acutely [9]. Data regarding the association between lopinavir concentrations and glucose metabolism are lacking.

Our study has several limitations. First, it is a cross sectional study and we therefore cannot compare lipid or glucose concentrations prior to lopinavir treatment. Second, patients with known diabetes or dyslipidaemia were excluded from the study, and it is possible that lopinavir may have exacerbated a pre-existing metabolic defect. Third, our sample size is relatively small, however, it is larger than most of the previous studies that have investigated this association $[11,17,19]$. We aimed to examine 50 participants to detect a correlation of 0.375. Our analyses used simple and multivariate regression analyses with various predictors, therefore we continued to slowly recruit eligible participants until the end of the study. Fourth, we investigated associations with pre-dose lopinavir concentrations and metabolic parameters. The pharmacokinetic parameters area under the curve or average steady state would be a better measure of overall drug exposure. Lastly, the last dose of lopinavir was not observed. To minimize recall bias, participants were requested to record the time of last dose on the appointment card for the day before pharmacokinetic sampling.

In conclusion, we did not find an association between lopinavir concentrations and lipid and glucose concentrations. Larger prospective studies are needed to establish whether an association exists between lopinavir concentrations and increasing lipids or glucose metabolism changes.

\section{Competing interests}

The authors declare that they have no competing interests.

\section{Authors' contributions}

PZS participated in the study design, acquisition of data, data analysis and interpretation and drafted the manuscript. HMM participated in the study design, data interpretation, and critically revised the manuscript. PJS performed the analysis of the samples and helped to draft the manuscript.
JAD participated in the study design, acquisition of data, and critically revised the manuscript. NSL participated in study design and critically revised the manuscript. GM conceived of the study, participated in study design, data interpretation and critically revised the manuscript. All authors read and approved the final manuscript.

\section{Acknowledgements}

The authors are grateful to Ms Carmen Delport (study coordinator) and her team, for the help with collecting blood samples; Ms Alicia Evans and pharmacology laboratory team for the sample preparation and analysis; and to the patients for their participation in this study.

This study was funded by the World Diabetes Foundation, South African Department of Health and the South African Medical Research Council, Clinical Infectious Diseases Research Initiative, and Discovery Health. The funding bodies had no role in study design; in collection, analysis and interpretation of the data; in writing of the manuscript; and in the decision to submit the manuscript for publication.

These data were presented in part at the 12th International Workshop on Adverse Drug Reactions and Co-morbidities in HIV, London, UK, 5 November 2010 (Abstract ADRLH-48). The abstract was also published in Antiviral Therapy 2010; 15 Suppl 4: A61 (abstract no P32).

\section{Author details}

'Department of Medicine, Division of Clinical Pharmacology, University of Cape Town, Cape Town, South Africa. ${ }^{2}$ Department of Medicine, Division of Endocrinology and Diabetes, University of Cape Town, Cape Town, South Africa.

Received: 15 May 2012 Accepted: 23 October 2012

Published: 26 October 2012

\section{References}

1. World Health Organization: Rapid advice antiretroviral therapy for HIV infection in adults and adolescents. World Health Organization; 2009. downloaded from http://www.who.int/hiv/pub/arv/rapid_advice_art.pdf on 03 August 2011

2. De Wit S, Sabin CA, Weber R, Worm SW, Reiss P, Cazanave C, El-Sadr C, Monforte W, Fontas A, Law E, Friis-Møller MG, Phillips N: Incidence and risk factors for new onset diabetes in HIV-infected patients. The data collection on adverse events of anti-HIV drugs (D: A: D) study. Diabetes Care 2008, 31:1224-1229.

3. Montes ML, Pulido F, Barros C, Condes E, Rubio R, Cepeda C, Dronda F, Antela A, Sanz J, Navas E, Miralles P, Berenguer J, Pérez S, Zapata A, González-García JJ, Peña JM, Vázquez JJ, Arribas JR: Lipid disorders in antiretroviral-naive patients treated with lopinavir/ritonavir-based HAART: frequency, characterization and risk factors. JAC 2005, 55:800804.

4. Anastos K, Lu D, Shi Q, Tien PC, Kaplan RC, Hessol NA, Cole S, Vigen C, Cohen M, Young M, Justman J: Association of serum lipid levels with HIV serostatus, specific antiretroviral agents, and treatment regimens. J Acquir Immune Defic Syndr 2007, 45:34-42.

5. Carr A, Samaras K, Thorisdottir A, Kaufmann GR, Chisholm DJ, Cooper DA: Diagnosis, prediction and natural course of HIV-1 protease-inhibitor associated lipodystrophy hyperlipidaemia, and diabetes mellitus: a cohort study. Lancet 1999, 353:2093-2099.

6. Galindo MJ, Verdejo J, Gonzalez-Mun M, Ferrer A, Polo R: Metabolic changes in protease inhibitor-naïve patients with lopinavir/ritonavir. J Acquir Immune Defic Syndr 2008, 48:628-629.

7. Dube' MP, Shen C, Greenwald M, Mather KJ: No impairment of endothelial function or insulin sensitivity with 4 weeks of the HIV protease inhibitors atazanavir or lopinavir-ritonavir in healthy subjects without HIV infection: A placebo-controlled trial. Clinical Infectious Disease 2008, 47:567-574.

8. Pao VY, Lee GA, Taylor S, Aweeka FT, Schwarz JM, Mulligan K, Schambelan M, Grunfeld C: The protease inhibitor combination lopinavir/ritonavir does not decrease insulin secretion in healthy, HIV-seronegative volunteers. AIDS 2010, 24:265-270.

9. Lee GA, Lo JC, Aweeka F, Schwarz JM, Mulligan K, Schambelan M, Grunfeld C: Single-dose lopinavir acutely inhibits insulin-mediated glucose disposal in healthy volunteers. Clin Infect Dis 2006, 43:658-660. 
10. de González Requena D, Blanco F, Garcia-Benayas T, et al: Correlation between lopinavir plasma levels and lipid abnormalities in patients taking Lopinavir/ritonavir. AIDS Patient Care STDS 2003, 17:443-445.

11. Gutiérrez F, Padilla S, Navarro A, Masiá M, Hernández I, Ramos J, Esteban A Martin-Hidalgo A: Lopinavir plasma concentrations and changes in lipid levels during salvage therapy with lopinavir/ritonavir-containing regimens. J Acquir Immune Defic Syndr 2003, 33:594-600.

12. León A, Martinez E, Sarasa M, López Y, Mallolas J, De Lazzari E, Laguno M, Milincovic A, Blanco JL, Larrousse M, Lonca M, Gatell JM: Impact of steadystate lopinavir plasma levels on plasma lipids and body composition after 24 weeks of Lopinavir containing therapy free of thymidine analogues. J Antimicrob Chemother 2007, 60:824-830.

13. Ren Y, Nuttall JJ, Egbers C, Eley BS, Meyers TM, Smith PJ, Maartens G, Mclleron HM: Effect of rifampicin on lopinavir pharmacokinetics in HIVinfected children with tuberculosis. J Acquir Immune Defic Syndr 2008, 47:566-569

14. American Diabetes Association: Diagnosis and classification of diabetes mellitus. Diabetes Care 2008, 31:S55-S60.

15. Grundy SM, Cleeman Jl, Merz CN, Brewer HB Jr, Clark LT, Hunninghake DB, Pasternak RC, Smith SC Jr, Stone NJ: Implications of recent clinical trials for the National Cholesterol Education Program Adult Treatment Panel III guidelines. Circulation 2004, 110:227-239.

16. ACTG adherence follow-up questionnaire; 2006. http://www.caps.ucsf.edu/ tools/surveys/pdf/2098.4188.pdf, on the 22 December 2006

17. Hurst M, Faulds D: Lopinavir. Drugs 2000, 60:1371-1379.

18. Bierman WF, van Vonderen MG, Veldkamp Al, Burger DM, Danner SA, Reiss $P$, van Agtmael MA: The lopinavir/ritonavir-associated rise in lipids is not related to lopinavir or ritonavir plasma concentration. Antivir Ther 2011, 16:647-655.

19. Decloedt EH, Mcllleron H, Smith P, Merry C, Orrell C, Maartens G: Pharmacokinetics of lopinavir in HIV-infected adults receiving rifampicin with adjusted doses of lopinavir-ritonavir tablets. AAC 2011, 55:3195-3200.

20. Bazzoli C, Jullien V, Le Tiec C, Rey E, Taburet AM: Intracellular pharmacokinetics of antiretroviral drugs in HIV infected patients, and their correlation with drug action. Clin Pharmacokinet 2010, 49:17-45.

21. Carr A, Samaras K, Chisholm DJ, Cooper D: Pathogenesis of HIV-1-protease inhibitor associated peripheral lipodystrophy, hyperlipidaemia and insulin resistance. Lancet 1998, 351:1881-1883.

22. Bongiovanni M, Bini T, Cicconi P, Landonio S, Meraviglia P, Testa L, Di Biagio A, Chiesa E, Tordato F, Biasi P, Adorni F, Monforte AD: Predictive factors of hyperlipidemia in HIV-infected subjects receiving lopinavir/ritonavir. AIDS Res Hum Retroviruses 2006, 22:132-138.

23. Tarr P, Telenti A: Toxigenetics of antiretroviral therapy: genetic factors that contribute to metabolic complications. Antivir Ther 2007, 12:999-1013.

24. Rhee MS, Hellinger JA, Sheble-Hall S, Cohen CJ, Greenblatt DJ: Relationship between plasma protease inhibitor concentrations and lipid elevations in HIV patients on a double-boosted protease inhibitor regimen (saquinavir/lopinavir/ritonavir). J Clin Pharmacol 2010, 50:392-400.

25. Ter Hostede HJM, Koopmans PP, Burger DM, Sprenger HG, Napel CT, Vriesendorp V, Richter C: Lopinavir plasma concentrations and serum lipids in therapy naïve HIV-patients: A sub- analysis of the FREE study. Pharmacology \& Pharmacy 2012, 3:90-96.

\section{Submit your next manuscript to BioMed Central and take full advantage of:}

- Convenient online submission

- Thorough peer review

- No space constraints or color figure charges

- Immediate publication on acceptance

- Inclusion in PubMed, CAS, Scopus and Google Scholar

- Research which is freely available for redistribution

Submit your manuscript at www.biomedcentral.com/submit
Ciomed Central 